\title{
First-principles calculations of niobium hydride formation in superconducting radio-frequency cavities
}

\author{
Denise C Ford, ${ }^{1,2}$ Lance D Cooley, ${ }^{2}$ David N Seidman ${ }^{3,4}$ \\ ${ }^{1}$ Department of Chemical and Biological Engineering, Northwestern University, \\ Evanston, IL 60208 USA \\ ${ }^{2}$ Superconducting Materials Department, Technical Division, Fermi National \\ Accelerator Laboratory, Batavia, IL 60510 USA \\ ${ }^{3}$ Department of Materials Science and Engineering, Northwestern University, \\ Evanston, IL 60208 USA \\ ${ }^{4}$ Northwestern University Center for Atom-Probe Tomography (NUCAPT), \\ Evanston, IL 60208 USA \\ Email: deniseford@u.northwestern.edu
}

\begin{abstract}
Niobium hydride is suspected to be a major contributor to degradation of the quality factor of niobium superconducting radio-frequency (SRF) cavities. In this study, we connect the fundamental properties of hydrogen in niobium to SRF cavity performance and processing. We modeled several of the niobium hydride phases relevant to SRF cavities and present their thermodynamic, electronic, and geometric properties determined from calculations based on density-functional theory. We find that the absorption of hydrogen from the gas phase into niobium is exothermic and hydrogen becomes somewhat anionic. The absorption of hydrogen by niobium lattice vacancies is strongly preferred over absorption into interstitial sites. A single vacancy can accommodate six hydrogen atoms in the symmetrically equivalent lowest-energy sites and additional hydrogen in the nearby interstitial sites affected by the strain field: this indicates that a vacancy can serve as a nucleation center for hydride phase formation. Small hydride precipitates may then occur near lattice vacancies upon cooling. Vacancy clusters and extended defects should also be enriched in hydrogen, potentially resulting in extended hydride phase regions upon cooling. We also assess the phase changes in the niobium-hydrogen system based on charge transfer between niobium and hydrogen, the strain field associated with interstitial hydrogen, and the geometry of the hydride phases. The results of this study stress the importance of not only the hydrogen content in niobium, but also the recovery state of niobium for the performance of SRF cavities.
\end{abstract}




\section{Introduction}

The niobium-hydrogen system has garnered great scientific interest over the past century as a representative system for metal-hydride phenomena in an industrially important material. Niobium is highly resistant to corrosion in both basic and acidic environments, and for this reason it is a material of choice for applications such as medical implants, reactor piping, and jewelry. Niobium, however, easily absorbs hydrogen if its protective oxide is compromised [1], which can significantly impact its properties. Hydrogen reduces the stability of niobium in corrosive media, either via local charge transfer or via elastic strain [2]. Hydride formation is central to the mechanism of hydrogen embrittlement [3-8], which affects the durability of steam piping and reactor membranes. Bieler et al. [9] noted that among the body-centered cubic (b.c.c.) metals, niobium exhibits unusual compliance for the standard crystallographic slip systems, creating a situation where dislocations are more stable when compared with other b.c.c. metals. Hydrogen atoms can effectively pin dislocations and other defects [10], and it is likely that dislocation migration involves dragging a Cottrell atmosphere of hydrogen atoms, along with its elastic strain field, near room temperature [11].

Of particular concern in the field of accelerator technology are the consequences of dissolved hydrogen and niobium hydride phases on niobium's superconducting properties. Pure niobium is the material of choice for superconducting radio-frequency (SRF) cavities, an enabling technology for high-performance linear particle accelerators, because it has the highest critical temperature $\left(T_{c}\right)$ of all the pure elements $(9.25 \mathrm{~K})$ and it is highly malleable, so that it can be formed into the complex shapes required for this technology. The incorporation of impurity atoms into niobium can cause a significant reduction of $\mathrm{T}_{\mathrm{c}}$, which has been demonstrated for hydrogen dissolved interstitially in b.c.c. niobium [12]. Furthermore, several niobium hydride phases are not superconducting above 1-2 K [13-15].

Unfortunately, the current forming and processing procedures for niobium SRF cavities afford many opportunities for hydrogen to be absorbed. The binding of hydrogen atoms to niobium lattice defects and grain boundaries serves as a potential reservoir of hydrogen [16]; and in polycrystalline sheets that have undergone cold-working steps, the dislocations and grain boundaries are conduits for hydrogen to the bulk interior, despite the existence of a native oxide coating. Once inside of the niobium, hydrogen can become trapped near the surface due to the elastic strain field [17-25]. It is therefore not surprising that hydrogen concentrations can be substantial in the near-surface region, to $\sim 100 \mathrm{~nm}$ deep, which is relevant to cavity performance. Concentrations of near 50 at.\% of hydrogen in niobium samples were recently reported by Y.-J. Kim and D. N. Seidman [26, 27] using atom probe tomography. Ciovati et al. [28] measured the hydrogen content of niobium samples using secondary ion mass spectrometry (SIMS) and report large counts of hydrogen, but were not able to determine a quantitative concentration because the hydrogen diffusivity was too large for calibration. They were able to determine, however, that the hydrogen content was reduced upon heat treatment, and the reduction was correlated with improved cavity performance. In contrast, Romanenko and Goncharova [29], using elastic recoil detection, found 10-20 at.\% hydrogen in the top $100 \AA$ of the niobium samples they studied, and no significant concentration correlation with processing treatments. Since some of their samples were extracted from cavities with different behavior, they suggested that the form in which hydrogen is present, and not the concentration of hydrogen, might therefore be responsible for differences in cavity performance.

The phases that hydrogen forms with niobium have been studied extensively experimentally. The phase diagram, given in a literature review by Manchester and Pitre [30] and summarized by Ricker and Myneni [1], is displayed in Figure 1. Disordered interstitial hydrogen in b.c.c. niobium is denoted $\alpha$ (for $\mathrm{H}$ concentrations $<24$ at. \%) and $\alpha^{\prime}$ (for greater $\mathrm{H}$ concentrations). 
Ordered interstitial hydrogen in face-centered niobium occurs at higher $\mathrm{H}$ concentrations and lower temperatures, and consists of the $\beta$ (orthorhombic, $\mathrm{H} / \mathrm{Nb} \approx 1$ ), $\delta$ (cubic, $\mathrm{H} / \mathrm{Nb} \approx 2$ ), and $\varepsilon$ (orthorhombic, $\mathrm{H} / \mathrm{Nb} \approx 3 / 4$ ) phases. The phases, $\lambda$ and $\lambda_{\mathrm{c}}$, and others, which are indicated by dashed lines, are not well established. It is possible for multiple hydride phases to occur simultaneously [31].

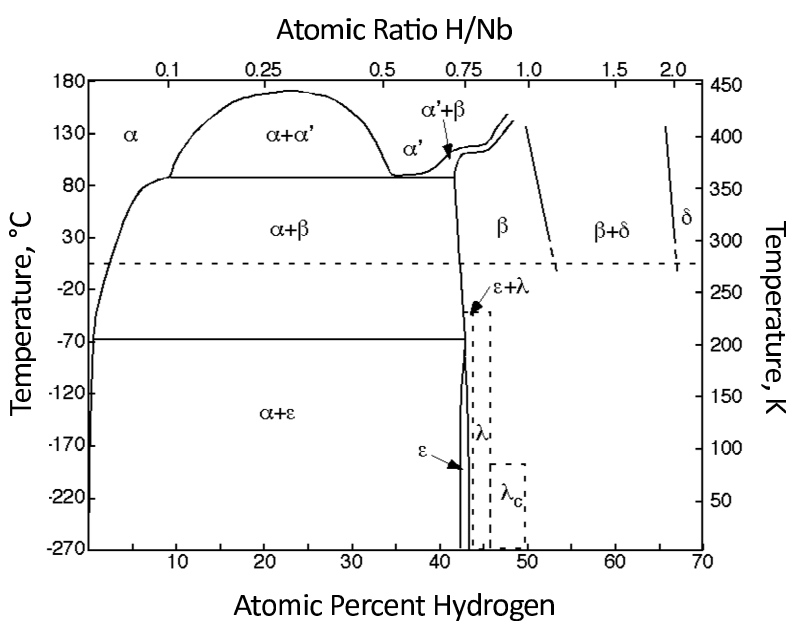

Figure 1. Nb-H phase diagram [30]. The $\alpha$ and $\alpha$ ' niobium hydride phases consist of interstitial hydrogen atoms dispersed in body-centered cubic (b.c.c.) niobium at differing low hydrogen concentrations, the $\beta$ phase consists of face-centered orthorhombic (f.c.o.) niobium with ordered hydrogen interstitials and with an $\sim \mathrm{NbH}$ stoichiometry, the $\delta$ phase has the fluorite structure with hydrogen in the tetrahedral sites of face-centered cubic (f.c.c.) niobium and an $\sim \mathrm{NbH}_{2}$ stoichiometry, the $\varepsilon$ phase is structured like the $\beta$ phase with the $\sim \mathrm{Nb}_{4} \mathrm{H}_{3}$ stoichiometry, and $\lambda$ and $\lambda_{\mathrm{c}}$ phases are experimentally unconfirmed niobium hydride phases.

Several factors, aside from temperature and concentration, can affect the formation of hydrides. As discussed in a review by Khaldeev and Gogel [2], dissolved hydrogen atoms can become trapped by several mechanisms: (1) the elastic strain that they impart to the niobium lattice upon absorption, known as self-trapping; (2) interaction with other dissolved impurities; and (3) absorption into lattice imperfects - point, line and planar. Trapping enthalpies and their effects on hydrogen diffusion have been evaluated experimentally, and theoretical models have been constructed to include elastic, electronic, and tunneling contributions - a book by Fukai [32] provides detailed information about these topics as well as references to many prior studies. It is difficult, however, to separate the effects of self, impurity, and defect trapping in physical experiments; and fundamental pieces of information about the behavior of hydrogen in niobium is still missing. Furthermore, extension of fundamental hydrogen behavior in niobium to SRF cavity processing is very challenging.

It is well known that hydride precipitates disrupt local superconductivity [33, 34]. While small niobium hydride precipitates should be weakly superconducting due to the proximity effect [35] when they are embedded in a niobium matrix; they are nonetheless problematic under RF operating conditions because very strong currents, of order $\mathrm{H}_{\mathrm{RF}} / \lambda$, are induced next to the cavity wall. Here $\mathrm{H}_{\mathrm{RF}}$ is the RF magnetic field amplitude and $\lambda$ is the magnetic penetration depth. Strong RF dissipation then occurs when the depairing current density for these coupled precipitates is exceeded [36]. For niobium the RF flux density, $\mu_{0} \mathrm{H}_{\mathrm{RF}}$ where $\mu_{0}$ is the permeability of free space, can be $>100 \mathrm{mT}$ at the equatorial regions of SRF cavities and $\lambda$ is $\sim 40$ $\mathrm{nm}$. Hence, the surface currents decay with depth from $\sim 10^{12} \AA \mathrm{m}^{-2}$. A drop in the cavity quality 
factor, $\mathrm{Q}$, as $\mathrm{H}_{\mathrm{RF}}$ increases is commonly observed (called $\mathrm{Q}$ drop), to which the onset of dissipation by small hydride precipitates may be a chief contributor. Recently, Romanenko et al. [37] observed the presence of hydride precipitates in SRF cavity regions that began to warm when $\mu_{0} \mathrm{H}_{\mathrm{RF}}$ exceeded $100 \mathrm{mT}$, whereas regions that did not exhibit heating also did not exhibit precipitates.

Large precipitates gain little or no benefit from the proximity effect, and produce dissipation at the onset of the RF magnetic field. The maximum size of a precipitate that can benefit from the proximity effect is determined by the coherence length, $\xi$, in the precipitate. Since the hydride phases are metallic, $\xi=\left(h v_{F}\right) /\left(2 \pi k_{B} T\right)$, where $h$ is Planck's constant, $v_{F}$ is the Fermi velocity, $k_{B}$ Boltzmann's constant, and $\mathrm{T}$ is the temperature in degrees Kelvin. Assuming $\mathrm{v}_{\mathrm{F}}$ is similar in niobium and niobium hydride, and the cavities are operated at $2 \mathrm{~K}$, we estimate a lower limit for the diameter of problematic precipitates to be $\sim 0.5 \mu \mathrm{m}$. SRF cavities that are heavily loaded with hydrogen (>2 wt. ppm [38]) commonly exhibit a fall-off of Q immediately with the application of $\mathrm{H}_{\mathrm{RF}}$ (called Q sickness or Q disease [39]). This may be due to large hydride precipitates.

In this article we present computational modeling of the $\alpha, \alpha^{\prime}, \beta$, and $\varepsilon$ phases of the niobiumhydrogen system, for which we have determined the phase properties from density functional theory (DFT) based calculations. We assess the roles of interstitial sites and $\mathrm{Nb}$ lattice vacancies for sequestering hydrogen, and discuss the nucleation of precipitates. We also examine the role of lattice strain, which may provide low energy pathways for hydrogen migration to newlyformed precipitates. We generalize our results to extended imperfections, which are computationally intense to model but are nonetheless important for SRF cavities.

\section{Methods}

All calculations were performed utilizing the Vienna Ab-initio Simulation Package (VASP) [40, 41], using DFT, periodic boundary conditions, and a plane wave basis set with a $400 \mathrm{eV}$ kinetic energy cutoff. The generalized gradient approximation (GGA) was used with the Perdew, Burke, Ernzerhof (PBE) exchange-correlation functional [42], and the core electrons were described by the projector-augmented-wave (PAW) pseudopotentials [43, 44]. All of the structure optimizations were calculated with all of the atom and cell degrees of freedom relaxed. Forces were converged to $0.02 \mathrm{eV} / \AA$. The geometry was optimized for each crystal structure with a 0.25 $\AA^{-1}$ gamma-centered Monkhorst-Pack [45] k-point mesh. The partial occupancies for the wavefunctions were set by the $1^{\text {st }}$ order Methfessel-Paxton method with a smearing width of 0.2 $\mathrm{eV}$. The Bader method [46-48] was used to assign charges to individual atoms.

The interactions between interstitial hydrogen atoms and the niobium lattice were assessed in $4 \times 4 \times 4$ unit cells (128 atoms) of b.c.c. niobium. The calculated lattice constant in the absence of impurity atoms is $3.32 \AA$, which is in good agreement with the experimental lattice constant of $3.30 \AA$ [49]. Tetrahedral and octahedral interstices were considered for impurity absorption. This unit cell was also used to assess the interactions between hydrogen atoms and a niobium lattice vacancy. The vacancy was created by removing one niobium atom, which corresponds to a vacancy concentration of $\sim 0.8$ at. $\%$. The calculated vacancy formation energy is $2.71 \mathrm{eV}$, which is in good agreement with the experimentally determined range of $2.6-3.1 \mathrm{eV}$ given in a review by Schultz and Ehrhart [50] and other first principles calculations [51, 52].

The niobium hydride structures were modeled as follows: $\beta$ ( $\mathrm{NbH}, 4$ formula units per unit cell), and $\varepsilon\left(\mathrm{Nb}_{4} \mathrm{H}_{3}-\mathrm{NbH}\right.$ with one $\mathrm{H}$ atom removed from the unit cell containing $4 \mathrm{Nb}$ and $4 \mathrm{H}$ atoms). Both the cccm and cmma structures were considered for $\mathrm{NbH}$ and the formation of $\mathrm{Nb}_{4} \mathrm{H}_{3}$. The lowest energy structures resulted from the $\mathrm{cccm} \mathrm{NbH}$ in both cases, and further analyses were 
only performed on these structures. The calculated lattice parameters for $\mathrm{NbH}$ are $3.50,4.88$, and $4.93 \AA$, which are within $\sim 1 \%$ of the experimental values [53]. The $\alpha$ and $\alpha$ ' niobium hydride phases were modeled as b.c.c. niobium (16 atoms per unit cell) with hydrogen atoms inserted into tetrahedral interstices. The properties for each concentration considered are reported as an average from two disordered configurations. An ordered configuration was also considered for the $\mathrm{Nb}_{16} \mathrm{H}_{16}$ structure, which did exhibit some significantly different properties from the disordered structures, and is presented in this article for comparison.

Several energetic properties are discussed in the results section for comparison between structures. Binding energies were calculated from various reference states, as were appropriate for the discussion, and these are specified in the results section. Self-trapping energies were calculated as the difference between the impurity configuration optimized in a relaxed niobium lattice versus that optimized in a rigid niobium lattice. Strain energies were calculated as the difference between the ideal b.c.c. niobium lattice and the lattice expanded by the impurity atom. Zero-point energy (ZPE) is included in all energy calculations, and was calculated from the formula $\mathrm{E}=0.5 \mathrm{~h} \Sigma v_{\mathrm{i}}$, where $\mathrm{h}$ is Planck's constant and $v_{\mathrm{i}}$ are the frequencies of the vibrational modes. The vibrational frequencies were determined from the Hessian matrix, which was created via a finite differences approach with a step size of $0.015 \AA$. The vibrational frequencies have been calculated for all of the atoms in the $\mathrm{Nb}_{16} \mathrm{H}_{\mathrm{x}}$ unit cells. The change in zero-point energy is approximated for unit cells containing 127 or 128 niobium atoms as resulting only from the difference in vibrations of the impurity atoms. For example, the zero point energy associated with the migration of $\mathrm{H}$ from an interstitial absorption site in b.c.c. niobium to an absorption site in a niobium lattice vacancy is calculated as $\mathrm{ZPE}\left(\mathrm{Nb}_{127} \mathrm{H}, \mathrm{H}\right.$ only relaxed $)-\mathrm{ZPE}\left(\mathrm{Nb}_{128} \mathrm{H}, \mathrm{H}\right.$ only relaxed).

\section{Results and Discussion}

\subsection{Dissolved hydrogen in body-centered cubic niobium}

Niobium absorbs hydrogen exothermically. The calculated absorption energy for hydrogen in tetrahedral sites is $-0.28 \mathrm{eV}$ relative to $\mathrm{H}_{2}$ in the gas phase and pure b.c.c. niobium, which is in good agreement with previous DFT calculations $[54,55]$ and the experimental heat of solution $[56,57]$. Hydrogen was found to be unstable in octahedral sites. Because niobium has a smaller Pauling electronegativity than hydrogen, 1.6 [58] and 2.2 [59], respectively, hydrogen becomes somewhat anionic when dissolved and expands the radius of a tetrahedral site from $0.52 \AA$ to 0.59 $\AA$. The lattice deformation causes 'self-trapping' of the hydrogen atom at strength of $0.18 \mathrm{eV}$. Hydrogen gains 0.65 e upon dissolution. The calculated absorption energy, charges, and lattice strain energies for the hydrogen are listed in table 1 and the absorption geometry is depicted in figure 2 .

Table 1. Charges and energetics of hydrogen in b.c.c. niobium tetrahedral interstices.

\begin{tabular}{lc}
\hline & $\mathrm{Nb}_{128} \mathrm{H}$ \\
\hline Charge on bonded $\mathrm{Nb}(\mathrm{e})$ & 0.14 \\
Charge on interstitial atom (e) & -0.65 \\
Absorption energy relative to $\mathrm{H}_{2, \text { gas }}(\mathrm{eV})$ & -0.28 \\
Lattice strain energy (eV) & 0.11 \\
\hline
\end{tabular}


a.

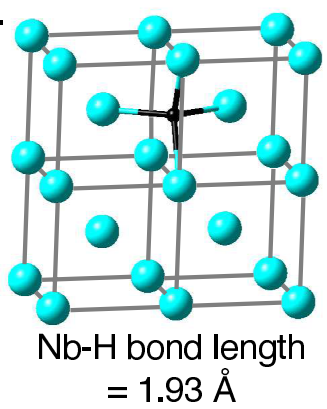

b.

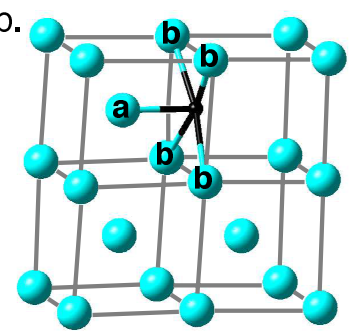

$\mathrm{Nb}_{\mathrm{a}}-\mathrm{H}$ bond length $=1.97 \AA$

$\mathrm{Nb}_{\mathrm{b}}-\mathrm{H}$ bond length $=2.27 \AA$

Figure 2. Geometry of hydrogen dissolved in a niobium tetrahedral interstice (a) and a niobium lattice vacancy (b). Small black spheres represent hydrogen and large blue spheres represent niobium.

Dissolved hydrogen atoms can be trapped by other hydrogen atoms as well as other interstitial impurities. Experimental studies have shown that one dissolved oxygen or nitrogen atom can trap one hydrogen atom in a nearby interstitial site at a strength of $\sim 0.1 \mathrm{eV} \mathrm{[60-62].} \mathrm{Since} \mathrm{dissolved}$ oxygen, nitrogen, and carbon may all be found in SRF niobium [63], we have assessed their interaction with dissolved hydrogen and the niobium lattice and present the results in another publication [64].

\subsection{Interaction between hydrogen and niobium lattice vacancies}

The charge distribution, absorption energy, and lattice strain energy caused by different amounts of hydrogen absorbed into a niobium lattice vacancy are given in table 2. The absorption geometry of one hydrogen atom in a niobium lattice vacancy is displayed in figure 2 , and is similar to octahedral binding with one niobium atom missing. The addition of one hydrogen atom to a vacant niobium lattice site causes a reduction in the charge on the hydrogen by $0.09 \mathrm{e}$ and change in bonding from four niobium atoms with a +0.14 e charge to four niobium atoms with a +0.13 e charge and one with a +0.11 e charge. As more hydrogen atoms are added to the vacancy, the charge on the hydrogen atoms is only slightly reduced; the charge, however, on the four niobium atoms with longer bonds to each hydrogen atom (type b, see figure 2) continues to increase. The lowest energy configuration consists of six hydrogen atoms, each filling one of the six symmetrically equivalent octahedral-like binding sites in the vacancy. Furthermore, we have found that hydrogen can continue to cluster around a vacancy after six hydrogen atoms have entered the vacancy at small energy gains (we attempted to add up to 6 additional hydrogen atoms), indicating that a niobium lattice vacancy can serve as a nucleation center for hydride phase formation.

Table 2. Charges on niobium atoms bound to hydrogen atoms and on the hydrogen atoms; absorption energies of hydrogen atoms in niobium lattice vacancies referred to the absorption energy of hydrogen atoms in tetrahedral interstitial sites; and lattice strain energies referred to the lattice strain energy of hydrogen atoms in tetrahedral interstitial sites. A vacancy binding site consists of one type a (charge given first in table) and four type b niobium atoms. See figure 2 for the definition of type $\mathrm{a}$ and $\mathrm{b}$ niobium atoms.

\begin{tabular}{lcccccc}
\hline & $\mathrm{Nb}_{128}$ & $\mathrm{Nb}_{122} \mathrm{H}$ & $\mathrm{Nb}_{127}$ & $\mathrm{Nb}_{127} \mathrm{H}$ & $\mathrm{Nb}_{127} \mathrm{H}_{4}$ & $\mathrm{Nb}_{127} \mathrm{H}_{6}$ \\
\hline Charge on bonded $\mathrm{Nb}(\mathrm{e})$ & $-0.01 / 0.01$ & 0.14 & 0.08 & $0.11 / 0.13$ & $0.08 / 0.26$ & $0.11 / 0.32$ \\
Charge on $\mathrm{H}(\mathrm{e})$ & - & -0.65 & - & -0.56 & -0.53 & -0.52 \\
Absorption energy per $\mathrm{H}(\mathrm{eV})$ & - & 0.00 & - & -0.41 & -0.39 & -0.34 \\
Lattice strain energy per $\mathrm{H}(\mathrm{eV})$ & - & 0.00 & - & -0.09 & -0.09 & -0.09 \\
\hline
\end{tabular}


Hydrogen occupancy in metal vacancies has been the subject of prior studies (see the review by Pundt and Kirchheim [65], and references therein). Predictions of six deuterium atoms occupying a niobium lattice vacancy with a binding energy of $-0.55 \mathrm{eV}$ each has been advanced by Nordlander et al. [66] based on effective medium theory calculations, and six hydrogen atoms with a binding energy of $-0.46 \mathrm{eV}$ each by Koike et al. [67] based on temperature, pressure, and concentration dependent resistivity measurements. Čížek and coworkers $[68,69]$ have performed several studies on hydrogen in niobium vacancies using positron annihilation, showing the existence of vacancies containing up to four hydrogen atoms; however, based on calculations at several levels of theory, they predicted that the mean-positron life-time in a one-vacancy-six hydrogen cluster would be nearly the same as in the bulk niobium, so they would not be able to detect this arrangement. Both Č́žzek et al. and Koike et al. measured an increase in vacancy concentration with increasing hydrogen concentration, although the hydrogen-induced vacancy concentration remained three orders of magnitude smaller than the total hydrogen concentration, and was comparable to the expected thermally-induced vacancy formation near the melting point. Rao, et al. showed a similar result based on DFT and statistical modeling [55]. The addition of hydrogen to metals including niobium is believed to induce vacancy formation, since several neighboring hydrogen atoms would be energetically stabilized by the presence of a vacancy [70, 71]. We find that the absorption energy of a single hydrogen atom into a vacancy is $0.41 \mathrm{eV}$ greater than into a tetrahedral interstitial site. The total calculated absorption energy of six hydrogen atoms into a lattice vacancy from tetrahedral interstices is $-2.05 \mathrm{eV}$, which would reduce the formation energy of a vacancy to $\sim 0.66 \mathrm{eV}$.

\subsection{Geometric and electronic aspects of niobium-hydride phase changes}

Figure 3 shows the energy difference between isolated interstitial hydrogen atoms occupying tetrahedral sites in b.c.c. niobium and the various niobium-hydride phases. These results highlight the thermodynamic favorability for the phase changes to occur at specific concentrations. The general shape of the solid-solution plot indicates that continual dissolution of hydrogen into niobium is favored with increasing hydrogen content up to a certain concentration at which the trend reverses. This behavior is common among transition metals and is related to a balance of the effects of metal lattice strain, hydrogen-metal attraction, and hydrogen-hydrogen repulsion [32]. A closer look at the plot reveals a distinct change in interaction energy at an atomic ratio of hydrogen to niobium $(\mathrm{H} / \mathrm{Nb})$ of 0.32 . Prior to this point the energy decrease was mild with increasing hydrogen concentration, and afterwards the energy difference increases more quickly. This correlates with a change in the elastic modulus, which signals the phase change from the $\alpha$ to the $\alpha$ ' phase. The addition of more hydrogen atoms continues to drive the energy decrease until an $\mathrm{H} / \mathrm{Nb}$ ratio of 0.75 is reached, at which point the ordered face-centered orthorhombic (f.c.o.) phases $\varepsilon$ and $\beta$ become energetically favored. While a configuration of ordered hydrogen in the tetrahedral interstices of b.c.c. niobium is plausible, this configuration is found to be higher in energy than that of f.c.o. $\beta$. 


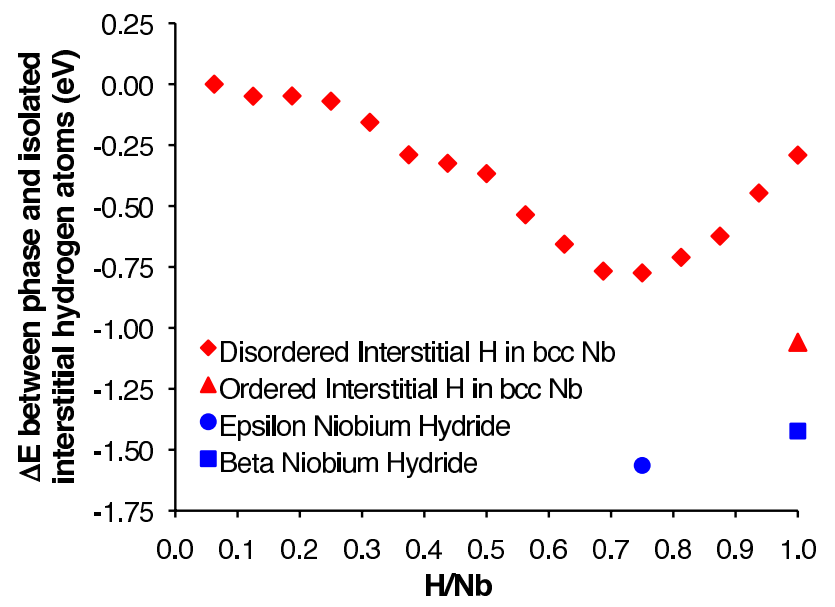

Figure 3. Energy difference between the niobium hydride phases and isolated hydrogen atoms in niobium tetrahedral interstices vs. hydrogen concentration.

The elastic deformation of the lattice with respect to hydrogen concentration in the solid-solution is shown in figure 4. The results are in good agreement with the temperature-dependent X-ray diffraction data of Albrecht et al. [72] at 0.10 and $0.54 \mathrm{H} / \mathrm{Nb}$ ratio. Albrecht et al., however, measure a smaller expansion at $0.25 \mathrm{H} / \mathrm{Nb}$, which is near the divide between the $\alpha$ and $\alpha$ ' regions. The known slope of the lattice parameter increase $(\Delta \mathrm{a} / \mathrm{a})$ versus $\mathrm{H} / \mathrm{Nb}$ plot $(0.058$ [73]) for the $\alpha$ phase is well-reproduced in this study (0.057). The deformation increases linearly in both solidsolution phases, but at a faster rate in the $\alpha$ than in the $\alpha^{\prime}$ phase.



Figure 4. Unit cell expansion (a is the lattice parameter) vs. hydrogen concentration. Least squares fits to the data are also given. The $\alpha$ and $\alpha$ ' phases are divided at $\sim 20$ at. $\%$, based on the division in the experimental phase diagram.

The importance of charge distribution for phase formation was suggested by Aboud and Wilcox [54], who studied the electronic charge state of hydrogen in niobium, vanadium, tantalum, palladium and palladium-niobium alloys utilizing DFT and Bader charge analysis. They found an increase in hydrogen's electronic charge, the host metal's lattice parameter, and the absorption energy for hydrogen into vanadium and tantalum for an increase in hydrogen concentration from 0.0625 to $0.125 \mathrm{H} /$ host atomic ratio; however, the electronic charge on hydrogen decreased when the hydrogen concentration was further increased to $0.25 \mathrm{H} /$ host ratio. The reduction in hydrogen 
charge was accompanied by an increase in absorption energy and host lattice parameter. When the concentration was increased to $0.5 \mathrm{H} /$ host ratio, they calculated a higher $\mathrm{H}$ charge and smaller absorption energy. The transition to the $\beta$ phase for vanadium and tantalum occurs at $0.5 \mathrm{H} / \mathrm{host}$ ratio. They explained their observations as competition between short-range repulsion between the dissolved hydrogen atoms and the attractive interaction between the hydrogen and host metal. Once short-range repulsion overtakes the attractive interaction a phase change will occur from solid solution to $\beta$ phase. They also suggested that minimizing the hydrogen charged is favored, but the data were not definitive. For niobium, they found the increase in hydrogen concentration caused an increase in hydrogen's electronic charge, niobium's lattice parameter, and the energy of absorption of hydrogen into niobium for all of the concentrations that they studied; however, they did not include results past the pure $\beta$ phase onset at $\mathrm{H} / \mathrm{Nb}$ ratio $\sim 0.7$.

We performed a Bader analysis on representative concentrations of hydrogen in niobium for the $\alpha, \alpha^{\prime}, \varepsilon$, and $\beta$ phases, and present the results in table 3 . The electronic charge on the hydrogen atoms, absorption energy, and self-trapping energy are all approximately the same for the two concentrations that we examined in the $\alpha$ phase, however the charge on the bonded niobium atoms varies within each configuration for $\mathrm{H} / \mathrm{Nb}$ atomic ratios greater than 0.0625 . The absorption and self-trapping energies both increase in the $\alpha$ ' phase, and the electronic charge on the hydrogen atoms varies within each configuration at higher concentrations. The average value for the $\alpha^{\prime}$ phase is $-0.66 \mathrm{e}$. The average electronic charge on the hydrogen atoms slightly decreases for the $0.75 \mathrm{H} / \mathrm{Nb}$ (b.c.c.) system to -0.65 e and further decreases to -0.64 e for 1.00 $\mathrm{H} / \mathrm{Nb}$ (b.c.c.).

Table 3. Charges on niobium atoms bound to hydrogen atoms and on the hydrogen atoms; absorption energies of hydrogen referred to b.c.c. niobium and gas phase hydrogen molecules; and self-trapping and lattice strain energies caused by the absorption of hydrogen.

\begin{tabular}{lcccccccc}
\hline & $\mathrm{Nb}_{16} \mathrm{H}$ & $\mathrm{Nb}_{16} \mathrm{H}_{3}$ & $\mathrm{Nb}_{16} \mathrm{H}_{8}$ & $\mathrm{Nb}_{16} \mathrm{H}_{12}$ & $\mathrm{Nb}_{16} \mathrm{H}_{16}$ & $\mathrm{Nb}_{16} \mathrm{H}_{16}-\mathrm{O}$ & $\mathrm{Nb}_{4} \mathrm{H}_{3}-\varepsilon$ & $\mathrm{NbH}-\beta$ \\
\hline $\begin{array}{l}\text { Charge on bonded } \\
\mathrm{Nb}(\mathrm{e})\end{array}$ & 0.13 & $0.07-0.32$ & $0.12-0.57$ & $0.36-0.69$ & $0.50-0.75$ & $0.64-0.71$ & 0.52 & 0.70 \\
Charge on H (e) & -0.66 & -0.66 & $-0.63-$ & $-0.62-$ & $-0.56-$ & -0.67 & $-0.67 /$ & -0.70 \\
$\begin{array}{l}\text { Absorption energy } \\
\text { per H (eV) }\end{array}$ & -0.24 & -0.26 & -0.29 & -0.31 & -0.26 & -0.31 & -0.37 & -0.33 \\
$\begin{array}{l}\text { Self-trapping energy } \\
\text { per H (eV) }\end{array}$ & -0.18 & -0.18 & -0.22 & -0.25 & -0.31 & -0.27 & -0.01 & 0.00 \\
$\begin{array}{l}\text { Strain energy per H } \\
(\mathrm{eV})\end{array}$ & 0.11 & 0.12 & 0.14 & 0.16 & 0.18 & 0.17 & 0.15 & 0.18 \\
\hline
\end{tabular}

Consideration of the f.c.o. $\varepsilon$ and $\beta$ phases allows for a greater $H$ charge than the b.c.c. phases at the same concentrations, and the charges on the niobium atoms do not vary within the $\varepsilon$ or $\beta$ phases. The calculated lattice strain energies are also given in table 3 and show that the strain is similar in the f.c.o. and b.c.c. phases at the same hydrogen concentrations; therefore the transition to the f.c.o. phases occurs to maximize the electronic interaction between the niobium and hydrogen constituents. This assertion is confirmed by the fact that the $\mathrm{NbH}$ configuration with ordered hydrogen atoms in b.c.c. niobium has a greater absorption energy and hydrogen charge than the configuration with disordered hydrogen atoms, but both of these properties are smaller in magnitude than they are for the f.c.o. ( $\beta$ phase) configuration. Additionally, the shortest $\mathrm{Nb}-\mathrm{H}$ bond in the $\beta$ phase is $1.94 \AA$, which is shorter than the shortest $\mathrm{Nb}-\mathrm{H}$ bond in the ordered configuration of hydrogen in b.c.c. niobium (1.95 $\AA$ ). Hydrogen-hydrogen repulsion is also 
reduced in the $\beta$ phase compared to the ordered configuration of hydrogen in b.c.c. niobium, as the nearest neighbor $\mathrm{H}-\mathrm{H}$ distances are $2.47 \AA$ and $2.42 \AA$, respectively.

\subsection{Application to niobium superconducting radio frequency cavities}

SRF cavities made from pure niobium undergo an elaborate fabrication and processing regimen to optimize the cavity quality factor and the accelerating gradient. Beginning with the ingot, forging and rolling occur, which create vacancies and dislocations that can sequester hydrogen. After $\mathrm{Nb}$ sheets are rolled, they are annealed at $700-1000{ }^{\circ} \mathrm{C}$ to recrystallize the niobium and degas the dissolved hydrogen, but then the half-cells are plastically deep-drawn, which reintroduces lattice imperfections. The half-cells are then welded together, which creates a further damaged zone near the weld bead. Chemical or electrochemical polishing with strong acids is applied to remove the surface layer that was damaged from forming. Not only do these chemical processes provide a source of hydrogen, they also remove the protective oxide coating on the niobium, affording the possibility for hydrogen to dissolve in it. This has been recognized [74] and therefore a high-temperature high-vacuum anneal $\left(800{ }^{\circ} \mathrm{C}\right.$ for $2 \mathrm{hr}$ or $650{ }^{\circ} \mathrm{C}$ for $\left.10 \mathrm{hr}\right)$, is employed. But are cavities hydrogen or hydride free at this point? Hydrogen gas is detected in and removed from the furnace during heat treatment; however, various recent experimental studies (see introduction section) have shown that large hydrogen concentrations (10-50 at. \%) are still present in the near-surface region.

Niobium forms an oxide coating if exposed to air or water, so the cavity immediately reforms an oxide surface layer once it is removed from the acid bath. However, as explained in the introduction section, the hydrogen already absorbed into the niobium is attracted to the near surface region due to the lattice strain field. In this study, we have explained the effect of the strain field in the niobium lattice on the relative stability of niobium-hydrogen complexes. The strain field also strongly affects the diffusion of the hydrogen in niobium. Blomqvist, et al. [75] showed via ab initio molecular dynamics simulations that different activation energies characterize hydrogen diffusion in the regions $400-650 \mathrm{~K}, 650-1500 \mathrm{~K}$, and at temperatures greater than $1500 \mathrm{~K}$. They attributed this effect to a difference between the average $\mathrm{Nb}-\mathrm{H}$ bond length in each temperature regime, which leads to unbinding to different degrees. At temperatures below room temperature the hydrogen plus binding-site pair and the strain field are considered to be fully coupled, and at temperatures below $244 \mathrm{~K}$ phonon-assisted tunneling dominates hydrogen diffusion [76]. The strain field also correlates with hydrogen concentration, as demonstrated by nuclear magnetic resonance [77] and Gorsky effect [78] studies, which display increasing activation energies for hydrogen diffusion in the $\alpha$ and $\alpha$ ' phases with increasing hydrogen concentration. Our calculations support these observations because we find an increase in strain energy with increasing concentration, and we also find an increase in the self-trapping energy per hydrogen atom. All of these observations imply that as the hydrogen concentration increases or temperature decreases, hydrogen's diffusivity decreases. Imperfections in the niobium lattice such as vacancies and dislocations, however, expand sites in the niobium lattice, thereby proving sites with lower energy barriers for hydrogen to hop between. This increases hydrogen's diffusivity and provides pathways for hydrogen atoms to find each other and form precipitated phases. Based on these assessments, two changes to the SRF cavity processing procedure might be considered: A cold acid polishing process, which might be selfarresting for hydrogen uptake, and annealing cavities prior to heavy chemical polishing, which will eliminate many of the defects that can enhance hydrogen uptake.

Our results show that the trapping energy of hydrogen by lattice vacancies is significantly greater than the trapping energy of hydrogen by other dissolved hydrogen atoms, which suggests the favorability of heterogeneous nucleation of hydride phases over homogeneous nucleation. The relative binding strengths of hydrogen in niobium in the different situations examined in this 
study are depicted in figure 5. Since high temperature high vacuum de-gassing removes many of the dislocations and other imperfections introduced by metal forming, nucleation of large precipitates along extended defects should be rare. The reduction of nucleation centers for large precipitates may be an under-appreciated change that occurs during the de-gassing treatment and repair of Q disease. Only recently has the SRF community begun to consider the relief of lattice imperfections in cavity processing, and we suggest continued studies. For example, in situ measurements of hydride precipitate stoichiometry and size at different levels of recovery could prove to be very useful.

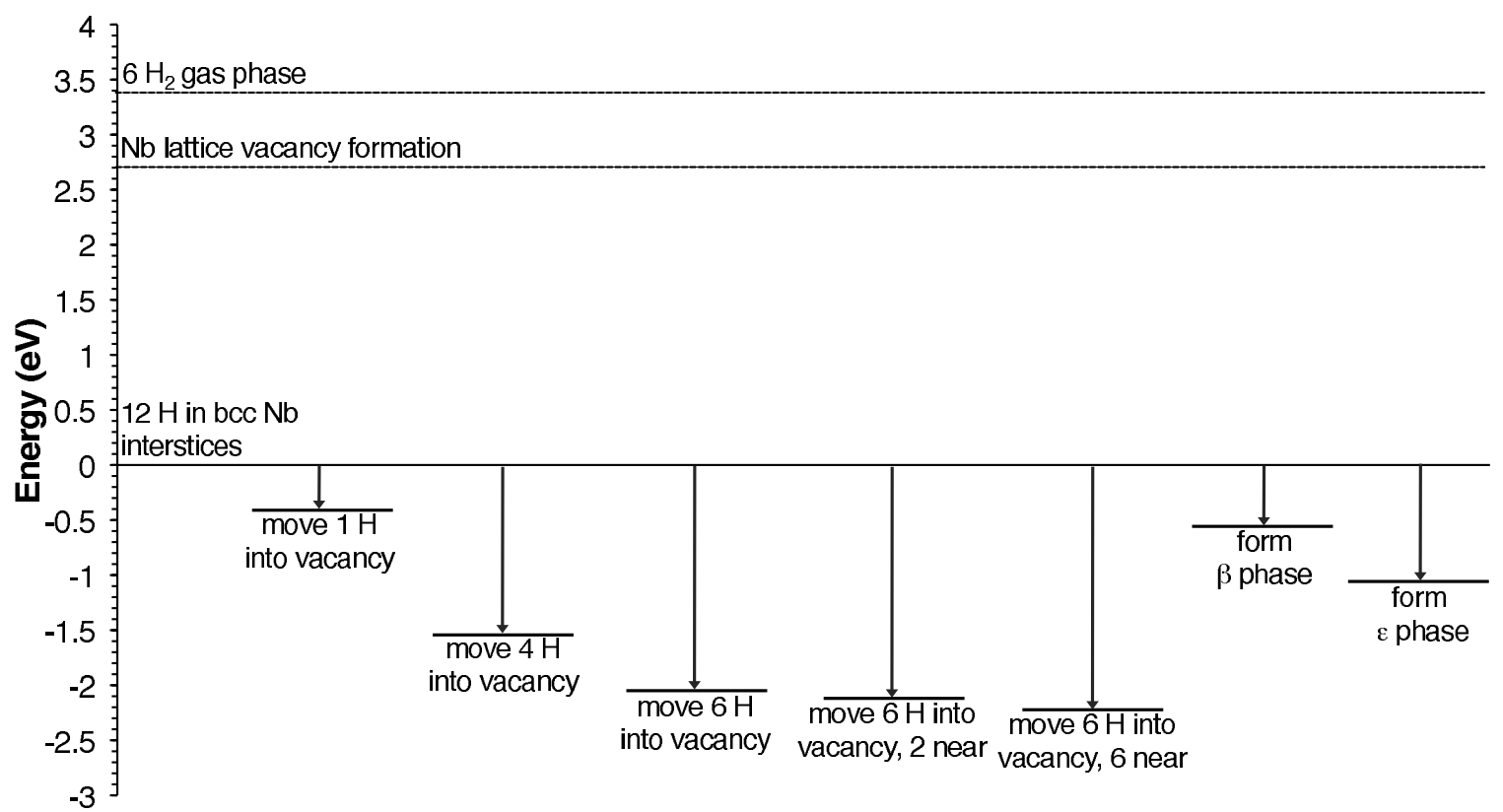

Figure 5. Energetic comparison of relevant configurations of hydrogen and niobium.

Finally, there are several reasons to eliminate the $\alpha^{\prime}$ phase, even in the absence of heterogeneous nucleation centers. First, we have shown the favorability in terms of the electronic interaction for the $\alpha$ ' phase to transform to the $\beta$ or $\varepsilon$ phase at high hydrogen concentrations. Second, Jisrawi et al. [12] demonstrated that $T_{c}$ decreases with increasing hydrogen concentration in the solidsolution phases, so the performance of the SRF cavity could still be affected in the absence of ordered hydride phases.

\section{Summary}

We have performed a density functional theory study of hydrogen in niobium. We evaluated hydrogen in interstitial lattice sites, hydrogen in niobium lattice vacancies, and the formation of the $\alpha, \alpha^{\prime}, \varepsilon$, and $\beta$ phases of niobium hydride. We discussed charge transfer from niobium to hydrogen (due to hydrogen becoming somewhat anionic in all cases), niobium lattice strain energy, and hydrogen-hydrogen repulsion as drivers for changes between phases. We compared homogenous to heterogeneous nucleation on lattice vacancies of ordered hydride phases. We found specifically:

- The absorption energy of hydrogen in niobium is $-0.28 \mathrm{eV}$, and hydrogen gains $0.65 \mathrm{e}$ upon absorption into a tetrahedral site. Hydrogen is not stable in octahedral sites in our model.

- A single vacancy in the bcc niobium crystal lattice is able to absorb significant amounts of hydrogen, being most stable for six hydrogen atoms, and the system energy continues 
to decrease with additional hydrogen atoms absorbing near the vacancy. Thus single lattice vacancies can serve as nucleation centers for hydride phases.

- Phase changes from disordered hydrogen in body-centered cubic niobium to ordered hydrogen in face-centered orthorhombic niobium occur to maximize the charge transfer from niobium to hydrogen and to minimize the repulsion between hydrogen atoms.

- Self-trapping energies increase with increasing hydrogen concentration, indicating that hydrogen diffusion is hindered with increasing hydrogen concentration.

The results were discussed in terms of the processing of niobium superconducting radiofrequency cavities. The tendencies to cluster hydrogen near niobium lattice vacancies (and, by extension, clusters of vacancies and extended lattice defects) drive the formation of hydride precipitates upon cooling a cavity in preparation for superconducting operation. Hydride precipitates can then disrupt the flow of RF currents and possibly result in the reduction of cavity quality factor. The importance of removing both hydrogen and hydride phase nucleation centers from the cavities to prevent Q disease and Q drop was stressed, and in situ experimental studies of precipitate size and structure at various stages of recovery were advised. Finally, we recommend consideration of two changes to the cavity processing procedure: using cold acid for chemical polishing and annealing the cavities prior to chemical treatment.

\section{Acknowledgements}

Fermilab is operated by the Fermi Research Alliance, LLC under Contract No. DE-AC0207CH11359 with the U.S. Department of Energy. We are pleased to acknowledge scientific discussions with A. Dzyuba, A. Romanenko, and J. Zasadzinski.

\section{References}

[1] Ricker R E and Myneni G R 2010 Evaluation of the propensity of niobium to absorb hydrogen during fabrication of superconducting radio frequency cavities for particle accelerators J. Res. Natl. Inst. Stand. Technol. 115 353-71

[2] Khaldeev G V and Gogel V K 1987 Physical and corrosion-electrochemical properties of the niobium hydrogen system Russ. Chem. Rev. 56

[3] Westlake D G 1969 ASM Trans. Quart. 62 1000-6

[4] Westlake D G 1969 Trans. Metall. Soc. AIME 245 287-92

[5] Grossbeck M L and Birnbaum H K 1977 Acta Metall. 25 135-47

[6] Gahr S, Grossbeck M L and Birnbaum H K 1977 Acta Metall. 25 125-34

[7] Gahr S and Birnbaum H K 1978 Acta Metall. 26 1781-8

[8] Owen C V and Scott T E 1972 Metall. Mater. Trans. B 3 1715-26

[9] Bieler T R, Wright N T, Pourboghrat F, Compton C, Hartwig K T, Baars D, Zamiri A, Chandrasekaran S, Darbandi P, Jiang H, Skoug E, Balachandran S, Ice G E and Liu W 2010 Phys. Rev. ST Accel. Beams 13031002

[10] Wilcox B A and Huggins R A 1960 J. Less Comm. Metals 2 292-303

[11] Szkopiak Z C 1968 Oxygen dependence of strain-ageing in niobium Acta Metall. 16381 91

[12] Jisrawi N M, Ruckman M W, Thurston T R, Reisfeld G, Weinert M, Strongin M and Gurvitch M 1998 Reversible depression in the Tc of thin Nb films due to enhanced hydrogen adsorption Phys. Rev. B 58 6585-91

[13] Isagawa $S 1980$ Hydrogen absorption and its effect on low-temperature electric properties of niobium J. Appl. Phys. 51 4461-70

[14] Ohlendorf D and Wicke E 1979 Heat capacities between 1.5 and $16 \mathrm{~K}$ and superconductivity of $\mathrm{V} / \mathrm{H}$ and $\mathrm{Nb} / \mathrm{H}$ alloys J. Phys. Chem. Solids $\mathbf{4 0}$ 721-8

[15] Welter J-M and Johnen F J 1977 Superconducting transition temperature and low temperature resistivity in the niobium-hydrogen system Z. Physik B 27 227-32 
[16] Myers S M, Baskes M I, Birnbaum H K, Corbett J W, DeLeo G G, Estreicher S K, Haller E E, Jena P, Johnson N M, Kirchheim R, Pearton S J and Stavola M J 1992 Hydrogen interactions with defects in crystalline solids Rev. Mod. Phys. 64 559-617

[17] Halbritter J 1987 On the oxidation and on the superconductivity of niobium Appl. Phys. A 43 1-28

[18] Romero A H, Schuller I K and Ramirez R 1998 Subsurface bonding of hydrogen in niobium: A molecular-dynamics study Phys. Rev. B 58 15904-8

[19] Lagos M, Martinez G and Schuller I K 1984 Kinetics of hydrogen absorption in transition metals and subsurface bonding Phys. Rev. B 29 5979-81

[20] Lagos M and Schuller I K 1984 Enhancement of hydgrogen uptake in metals and subsurface bonding Surf. Sci. 138 L161-7

[21] Li Y, Erskine J L and Diebold A C 1986 High-resolution electron-energy-loss spectroscopy of hydrogen chemisorption at $\mathrm{Nb}(100)$ surfaces: Evidence for subsurface absorption sites Phys. Rev. B 34 5951-4

[22] Fang B-S, Ballentine C A and Erskine J L 1987 Electronic properties of Nb and H-treated Nb surfaces Phys. Rev. B 36 7360-3

[23] Smith R J 1980 Characterization of the hydrogen adsorption/absorption process for $\mathrm{Nb}(110)$ with surface spectroscopy Phys. Rev. B 45 1277-80

[24] Smith R J 1980 Photoemission studies of hydrogen chemisorption on Nb Phys. Rev. B 21 3131-6

[25] Cabrera A L, Espinosa-Gangas J, Jonsson-Akerman J and Schuller I K 2002 Kinetics of subsurface hydrogen adsorbed on niobium: Thermal desorption studies J. Mater. Res. 17 2698-704

[26] Kim Y-J and Seidman D N Atom-scale analyses of Nb-hydrides via local-electrode atomprobe tomography Scripta Mater. sumbitted

[27] Kim Y-J and Seidman D N Atomic-scale chemical analyses of niobium-hydrides using atom-probe tomography and transmission electron microscopy Appl. Phys. Lett.

\section{submitted}

[28] Ciovati G, Myneni G, Stevie F, Maheshwari P and Griffis D 2010 High field Q slope and the baking effect: Review of recent experimental results and new data on $\mathrm{Nb}$ heat treatments Phys. Rev. ST Accel. Beams 13022002

[29] Romanenko A and Goncharova L V 2011 Elastic recoil detection studies of near-surface hydrogen in cavity-grade niobium Supercond. Sci.Tech. 24105017

[30] Manchester F D and Pitre J M 2000 (Materials Park, OH: ASM International)

[31] Kim Y-J, Seidman D N, Tao R and Klie R F Direct atomic-scale imaging of Nb-hydrides and oxides using atom-probe tomography and aberration-corrected STEM/EELS Nat. Mater. submitted

[32] Fukai Y 2005 The Metal-Hydrogen System (Berlin: Springer)

[33] Vinnikov L Y and Goluboe A O 1982 Direct observation of magnetic structure in niobium single crystals with hydride precipitate pinning centres Phys. Stat. Sol. 69 631-6

[34] Welling M S, Aegerter C M and Wijngaarden R J 2005 Self-organized criticality induced by quenched disorder: Experiments on flux avalanches in NbHx films Phys. Rev. B 71 104515

[35] de Gennes P G 1989 Superconductivity of Metal and Alloys (Redwood City, CA: Addison-Wesley)

[36] Gurevich A and Ciovati G 2008 Phys. Rev. B 77 104501-21

[37] Romanenko A, Wu G, Cooley L and Ciovati G 2011 Post-baking losses in electropolished niobium cavities: dissection studies. In: SRF2011, (Chicago, IL) THPO008 
[38] Knobloch J and Padamsee H 1997 Enhanced susceptibility of Nb cavity equator welds to the hydrogen related Q-virus. In: 1997 Workshop on RF Superconductivity, (Abano Terme, Italy) pp 434-9

[39] Knobloch J 2003 The "Q disease" in superconducting niobium RF cavities. In: Hydrogen in Materials \& Vacuum Systems: First International Workshop on Hydrogen in Materials and Vacuum Systems, ed G R Myneni and S Chattopadhyay (Melville, New York: AIP) pp 133-50

[40] Kresse G and Hafner J 1993 Abinitio molecular-dynamics for liquid-metals Phys. Rev. B 47 558-61

[41] Kresse G and Furthmuller J 1996 Efficient iterative schemes for ab initio total-energy calculations using a plane-wave basis set Phys. Rev. B 54 11169-86

[42] Perdew J P, Burke K and Ernzerhof M 1996 Phys. Rev. Lett. 773865

[43] Kresse G and Joubert D 1999 Phys. Rev. B 59 1758-1775

[44] Blöchl P E 1994 Phys. Rev. B 50 17953-17979

[45] Monkhorst H J and Pack J D 1976 Phys. Rev. B 13 5188-92

[46] Tang W, Sanville E and Henkelman G 2009 J. Phys. Condens. Mat. 21084204

[47] Sanville E, Kenny S D, Smith R and Henkelman G 2007 J. Comput. Chem. 28899

[48] Henkelman G, Arnaldsson A and Jónsson H 2006 Comput. Mater. Sci. 36254

[49] Huber K P and Herzberg G 1979 Molecular Spectra and Molecular Structure. IV. Constants of Diatomic Molecules (New York: Van Nostrand Reinhold Co.)

[50] 1991 Atomic Defects in Metals vol 25 (Berlin: Springer-Verlag)

[51] Korzhavyi P A, Abrikosov I A and Johansson B 1999 First-principles calculations of the vacancy formation energy in transition and noble metals Phys. Rev. B 59 11693-703

[52] Korhonen T, Puska M J and Nieminen R M 1995 Vacancy-formation energies for fcc and bcc transition metals Phys. Rev. B 51 9526-32

[53] Schober T, Pick M A and Wenzl H 1973 Electron microscopy of b-hydride in niobium Phys. Status Solidi A 18 175-82

[54] Aboud S and Wilcox J 2010 A density functional theory study of the charge state of hydrogen in metal hydrides J. Phys. Chem. C 114 10978-85

[55] Rao J-P, Ouyang C-Y, Lei M-S and Jiang F-Y 2011 Vacancy and H interactions in Nb Chin. Phys. Lett. 2812701

[56] Veleckis E and Edwards R K 1969 Thermodynamic properties in the systems vanadiumhydrogen, niobium-hydrogen, and tantalum-hydrogen J. Phys. Chem. 73 683-92

[57] Pryde J A and Titcomb C G 1972 Thermodynamic data of the hydrogen-niobium system J.Phys. C 5 1301-8

[58] Pauling L 1960 The Nature of the Chemical Bond (Cornell Univ., USA)

[59] Allred A L 1961 J.Inorg. Nucl. Chem. 17 215-21

[60] Pfeiffer G and Wipf H 1975 The Trapping of hydrogen in niobium by nitrogen interstitials J. Phys. F: Met. Phys. 6 167-79

[61] Shirley A I, Hall C K and Prince N J 1983 Trapping of hydrogen by oxygen and nitrogen impurities in niobium, vanadium and tantalum Acta Metall. 31 985-92

[62] Au J J and Birnbaum H K 1977 Acta Metall. 261105

[63] Singer W, Singer X and Wen H M 2003 Materiaux \& Techniques 91 13-8

[64] Ford D, Cooley L D and Seidman D N Suppression of hydride precipitates in niobium superconducting radio-frequency cavities Supercond.Sci.Tech. submitted

[65] Pundt A and Kirchheim R 2006 Hydrogen in metals: microstructural aspects Annu. Rev. Mater. Res. 36 555-608

[66] Nordlander P, Nørskov J K, Besenbacher F and Myers S M 1989 Multiple deuterium occupancy of vacancies in Pd and related metals Phys. Rev. B 40 1990-2

[67] Koike H, Shizuku Y, Yazaki A and Fukai Y 2004 Superabundant vacancy formation in $\mathrm{Nb}-\mathrm{H}$ alloys; resistometric studies J. Phys.: Condens. Matter 16 1335-49 
[68] Cizek J, Prochazka I, Becvar F, Kuzel R, Cieslar M, Brauer G, Anwand W, Kirchheim R and Pundt A 2004 Hydrogen-induced defects in bulk niobium Phys. Rev. B 69224106

[69] Cizek J, Prochazka I, Danis S, Brauer G, Anwand W, Gemma R, Nikitin E, Kirchheim R, Pundt A and Islamgaliev R K 2009 Hydrogen-vacancy complexes in electron-irradiated niobium Phys. Rev. B 79054108

[70] Fukai Y and Okuma N 1993 Japan J. Appl. Phys. 32 L1256

[71] Fukai Y and Okuma N 1994 Phys. Rev. Lett. 731640

[72] Albrecht W M, Goode W D and Mallett M W 1959 Reactions in the niobium-hydrogen system J. Electrochem. Soc. 106 981-6

[73] Peisl H 1978 Hydrogen in Metals I, Topics in Applied Physics vol 28 (Berlin: SpringerVerlag)

[74] Hoyt E W 1972 Niobium surfaces for Rf superconductors J. Vac. Sci. Technol. 9144

[75] Blomqvist A, Pálsson G K, Araújo C M, Ahuja R and Hjörvarsson B 2010 Significance of self-trapping on hydrogen diffusion Phys. Rev. Lett. 105185901

[76] Sundell P G and Wahnstrom G 2004 Self-trapping and diffusion of hydrogen in Nb and Ta from first principles Phy. Rev. B 70224301

[77] Mauger P, Williams W and Cotts R 1981 Diffusion and NMR spin lattice relaxation of $1 \mathrm{H}$ in alpha' TaHx and NbHx J. Phys. Chem. Solids 42 821-6

[78] Bauer H, Völkl J, Tretkowski J and Alefeld G 1978 Diffusion of hydrogen and deuterium in $\mathrm{Nb}$ and Ta at high concentration. Z. Physik B 29 17-26 